\title{
DETERMINATION OF THE AUGMENTATION TERMINAL FOR FINITE ABELIAN GROUPS
}

\author{
BY MICHAEL SINGER
}

Communicated by Olga Taussky Todd, May 20, 1977

Let $G$ be a finite abelian group, and let $I G$ denote the augmentation ideal in the integral group ring $\mathbf{Z G}$. The graded ring associated with the filtration on $\mathrm{ZG}$ determined by the powers of $I G$ is

$$
\text { gr } \mathrm{Z} G=\sum_{n \geq 0} \oplus I G^{n} \mid I G^{n+1}
$$

We write $Q_{n} G=I G^{n} / I G^{n+1}$. As is well known [1], [6], the sequence $Q_{n} G$ becomes stationary after a finite number of steps. We call its terminal value the augmentation terminal, $Q_{\infty} G$. We outline here a method for investigating $Q_{\infty} G$ for any $G$.

An obvious splitting allows us to assume that $G$ is a $p$-group.

We choose a generator for each cyclic direct factor of $G$. Let $\Gamma$ be our set of such generators, and let $\Lambda=\{\lambda \mid \lambda+1 \in \Gamma\}$. Generalizing Lemma 2 of [3] we have

LEMMA. For $n \geqslant 1$ the set of $n$-fold products of elements of $\Lambda$ generates $I G^{n} ;$ a fortiori it generates $Q_{n} G$.

If $\lambda \in \Lambda$ there is an integer $r$ such that $(\lambda+1)^{p^{r}}-1=0$. Furthermore, by the structure of $G$, these equations are the only possible source of relations among the elements of $\Lambda$. Hence we have immediately

THEOREM 1. Let $f\left(\lambda_{1}, \ldots, \lambda_{k}\right)$ be a nontrivial relator in $Q_{n} G$, where the $\lambda_{i} \in \Lambda$ Let $\lambda_{i}+1$ be of order $p^{r_{i}}$ in $G$, each $i$. Let $X_{1}, \ldots, X_{k}$ be indeterminates over $\mathrm{Z}$. Then there are polynomials $h_{i}\left(X_{1}, \ldots, X_{k}\right)$ with integer coefficients such that

$$
f\left(X_{1}, \ldots, X_{k}\right)-\sum_{i=1}\left\{\left(X_{i}+1\right)^{p^{r_{i}}}-1\right\} h_{i}\left(X_{1}, \ldots, X_{k}\right)
$$

has no terms of degree $\leqslant n+1$.

Actually using this result to find relators is far from easy, as the references show [2], [7], [8]. If $G$ is an elementary $p$-group we have

THEOREM 2. The relators in $Q_{\infty} G$ are generated by $\{p \lambda \mid \lambda \in \Lambda\}$ and $\left\{\lambda^{p} \mu-\lambda \mu^{p} \mid \lambda, \mu \in \Lambda\right\}$.

AMS (MOS) subject classifications (1970). Primary 20 C05. 
Proof. Easy hand calculation shows that these are indeed relators. It is trivial to work out the group given by these relators and then use Theorem 5 of [6] (see also Theorem 3 below).

By much more tedious calculation one can show

Lemma. If $\lambda, \mu \in \lambda$ and $\lambda+1, \mu+1$ have order $p^{2}$ in $G$, then $\lambda^{p^{2}} \mu^{p}-$ $\lambda^{p} \mu^{p^{2}}$ is a relator.

The appropriate generalization is readily conjectured, but a direct proof is likely to be very difficult.

We developed in [3], [7] and [8] a technique of "standard forms" which is generally suitable for determining the structure of $I G^{n}$ modulo a given set of relators of $Q_{n} G$. This technique may be applied to any $G$. If the order of the group so determined is the same as that of $Q_{\infty} G$, then $Q_{\infty} G$ has been found. Otherwise, another relator must be hunted down.

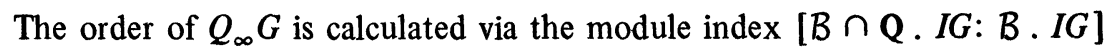
where $B$ is the maximal order in $Q G$. This follows from [6], where we used our results on invertible powers of ideals [4], [5]. Specifically, by direct calculation from Theorem 5 of [6].

THEOREM 3. Let $G$ be the direct product of $a_{i}$ cyclic groups of order $p^{i}$, $1 \leqslant i \leqslant m$. Then the order of $Q_{\infty} G$ is $p^{J}$, where

$$
p^{J}=p^{t_{1}}+\cdots+p^{t_{m-1}}+\frac{p^{t_{m}+1}-1}{p-1}
$$

in which

$$
t_{i}=a_{1}+2 a_{2}+\cdots+(i-1) a_{i-1}+i\left(a_{i}+\cdots+a_{m}-1\right), \quad 1 \leqslant i \leqslant m .
$$

\section{BIBLIOGRAPHY}

1. Franz Bachmann and Luzius Grünenfelder, The periodicity in the graded ring associated with an integral group ring, J. Pure Appl. Algebra 5 (1974), 253-264.

2. David Ford and Michael Singer, Relations in $Q_{n}\left(Z_{4} \times \mathrm{Z}_{8}\right)$ and $Q_{n}\left(\mathrm{z}_{8} \times \mathrm{Z}_{8}\right)$, Comm. Algebra 5 (1977), 83-86.

3. Michael Singer, On the graded ring associated with an integral group ring, Comm. Algebra 3 (1975), 1037-1049.

4. - Invertible powers of ideals over orders in commutative separable algebras, Proc. Cambridge Philos. Soc. 67 (1970), 237-242.

5. - An elementary proof of the invertible powers theorem, Proc. Cambridge Philos. Soc. 73 (1973), 289-291.

6. - On the augmentation terminal of a finite abelian group, J. Algebra 41 (1976), 196-201.

7. - Determination of the augmentation terminal for all finite abelian groups of exponent 4, Comm. Algebra 4 (1976), 639-645.

8. Determination of the augmentation terminal for all finite abelian groups of exponent 8, Comm. Algebra 5 (1977), 87-100.

DEPARTMENT OF MATHEMATICS, STANFORD UNIVERSITY, STANFORD, CALIFORNIA 94305 